\title{
Is the first day postoperative review necessary after cataract extraction?
}

\author{
A Tufail, A J E Foss, A M P Hamilton
}

\begin{abstract}
Background-In current ophthalmic practice day-case surgery cataract patients are conventionally discharged and then reviewed the following morning thus limiting the advantages of what 'true' day-case surgery strives to achieve. The aim of this study was to see if there was a difference in outcome between 'true' day-case cataract surgery and non-day-care surgery. Methods-A total of 387 consecutive cataract operations were followed, comprising 122 local anaesthetic day-cases, 149 local anaesthetic non-day-cases, 63 general anaesthetic non-day-cases, and 53 general anaesthetic day-cases.

Results-Although not randomised the groups were comparable with respect to age, operator grade, sex, presence of diabetes, anaesthetic type, pre and postoperative visual acuities, and time to first planned outpatient visit. There were 10 early postoperative complications in the day-case group $(5 \cdot 71 \%$ of total) and 14 in the non-day-case group (6.6\% of total), the commonest complications in both groups were raised intraocular pressure, corneal oedema, and wound leaks. One patient in each group had an early complication that necessitated attending the casualty department. The visual outcomes in both groups were comparable.

Conclusions-These findings suggest that there were no preventable complications within the constraints of the number of operations studied and that no additional risk is attached to 'true' day-case surgery relative to non-day-case surgery.

(Br f Ophthalmol 1995; 79: 646-648)
\end{abstract}

Conventionally patients are reviewed the day after cataract surgery in order to screen for early postoperative complications. There is little published documentation to justify this traditional practice. It is commonly accepted that the 1 day postoperative review is essential for eliciting the signs of early postoperative complications. This review extends to day-case patients who are either sent home or accommodated in hostels and then return for review

Moorfields Eye Hospital, City Road, London EC1V 2PD A Tufail

A J E Foss

A M P Hamilton

Correspondence to: Alexander Foss, Moorfields Eye Hospital, City Road, Eye Hospital, City Ro
London ECIV 2PD. London EC1V 2PD. 14 February 1995

Table 1 Proportion of cases undergoing local (LA) or general anaesthesia $(G A)$

\begin{tabular}{lcc}
\hline Anaesthetic type & True day-case & Non-day-case \\
\hline GA & 53 & 63 \\
LA & 122 & 149 \\
Total & 175 & 212 \\
\hline p $=0.52$ & &
\end{tabular}

the next day. It would be of benefit to both the patient and doctor if this return visit was shown not to be necessary to screen for early complications. We therefore decided to see if there was excess morbidity in patients undergoing 'true' day-case surgery (that is, patients discharged on the day of surgery with no review on the following day).

\section{Methods}

The outcome of 387 consecutive patients undergoing 'true' day-case cataract surgery and non-day-case surgery was recorded. All surgery was performed by a single consultant's team at Moorfields Eye Hospital. The allocation of a patient to either true day-case surgery or local versus general anaesthesia was not random but was decided on the patient's general health and social circumstances. Extracapsular cataract extraction and intraocular lens implant was the main method used; the other technique used was phakoemulsification. If a particular patient had both eyes operated on during the study period only one eye was included, the choice being random.

The true day-case patients were reviewed by a member of the surgical team 4 to 6 hours postoperatively. The non-day-cases were reviewed the following day. A standard postoperative regimen of dexamethasone and chloramphenicol drops was prescribed to each patient. There was a standard postoperative follow up appointment made for both groups of patients. The first postoperative visit was 7 or 10 days after the day of surgery. All postoperative complications up to and including the day of first postoperative visit were recorded. Any additional postoperative visits to the casualty department were noted. If a patient suffered a significant operative or immediate postoperative problem in the true day-case group the patient was kept in for review the following day.

\section{Results}

In the period of study there were operations on 175 eyes of true day-cases and 212 eyes of non-day-cases performed. The two groups

Table 2 Distribution of operator grade by 'true' day-case versus non-day-case cases

\begin{tabular}{lcc}
\hline Operator grade & True day-case & Non-day-case \\
\hline Consultant & 12 & 9 \\
Senior registrar & 91 & 114 \\
Registrar & 55 & 53 \\
Senior house officer & 17 & 36 \\
Total & 175 & 212 \\
\hline
\end{tabular}

$\chi^{2}$ for trend $=1 \cdot 74, p=0 \cdot 19$ 
Table 3 Proportion of diabetics in the 'true' day-case and non-day-case groups

\begin{tabular}{lcc}
\hline & True day-case & Non-day-case \\
\hline Non-diabetic & 134 & 168 \\
Diabetic & 41 & 44 \\
Total & 175 & 212 \\
\hline
\end{tabular}

were compared on a number of different variables. The age (day-case mean age $70 \cdot 6$, nonday-case 69.5), sex (day-case group 100 female and 75 male, non-day-case 114 and 98 respectively: $p=0.57$ ), anaesthetic type (Table 1 ), operator grade (Table 2), and the presence of diabetes (Table 3 ) did not show any significant difference (using the $\chi^{2}$ test and the $\chi^{2}$ test for trend as appropriate) between the two groups.

The preoperative corrected visual acuity (Table 4) showed no significant difference between the two groups $\left(\chi^{2}\right.$ test for trend $=0.099, p=0.80$ ), as did the final postrefraction visual acuity $\left(\chi^{2}\right.$ for trend $=0.056$, $\mathrm{p}=0 \cdot 81$ ) (Table 5).

There were 10 complications in the early postoperative period in the day-case group and 13 in the non-day-case group (Table 6). Raised intraocular pressure was taken as pressure that required treatment - that is, ocular pressures greater than $30 \mathrm{~mm} \mathrm{Hg}$. There were no intraocular pressures noted at the first postoperative outpatient visit that were not detected on discharge. Patients with complications who were originally designated as day-cases but were subsequently kept in overnight owing to intraoperative or immediate postoperative complications were counted as complications in the day-case group, and not included in the non-day-case group even though they were admitted overnight. All the wound leaks were detected before discharge in both groups although one iris prolapse was noted in each group on the first postoperative visit.

One patient in each group required an additional casualty visit before their first scheduled outpatient appointment. Both patients were complaining of ocular discomfort, the only relevant finding being moderate anterior uveitis in both cases. There were no cases of early endophthalmitis in either group although the non-day-case group had one case of endophthalmitis at 3 months postoperatively.

\section{Discussion}

The established 'correct' time of discharge has constantly been under review over the years ${ }^{1-3}$

Table 4 Distribution of visual acuity in the 'true' day-case and non-day-case groups

\begin{tabular}{lcc}
\hline Preoperative visual acuity & Day-case & Non-day-case \\
\hline $6 / 9$ & 1 & 0 \\
$6 / 12$ & 13 & 17 \\
$6 / 18$ & 19 & 24 \\
$6 / 24$ & 17 & 18 \\
$6 / 36$ & 30 & 41 \\
$6 / 60$ & 45 & 38 \\
Less than $6 / 60$ & 60 & 74 \\
Total & 175 & 212 \\
\hline
\end{tabular}

$\chi^{2}$ for trend $=0 \cdot 066, p=0 \cdot 80$.
Table 5 Distribution of visual outcome in the 'true' day-case and non-day-case groups

\begin{tabular}{lcc}
\hline Postoperative visual acuity & True day-case & Non-day-case \\
\hline $6 / 5$ & 4 & 8 \\
$6 / 6$ & 39 & 59 \\
$6 / 9$ & 60 & 68 \\
$6 / 12$ & 12 & 12 \\
$6 / 18$ & 2 & 3 \\
$6 / 24$ & 1 & 2 \\
$6 / 36$ & 2 & 3 \\
$6 / 60$ & 3 & 3 \\
Less than $6 / 60$ & 0 & 4 \\
Total & 123 & 162 \\
\hline
\end{tabular}

$\chi^{2}$ for trend $=0.056, p=0.81$.

and at each suggestion of reducing the admission time there have been protests on a mainly medicolegal basis. ${ }^{4}$ Established recent textbooks of ophthalmology mostly suggest that the patient must be reviewed on the day after cataract surgery. ${ }^{5}$ As discharging the patient on the day of surgery with no review the following day is not established practice it is important to see if there is any excess morbidity attached. The patients were not randomly allocated between the two groups as this would be impractical; many patients are forced to stay overnight because of medical factors or social circumstances. In spite of this the two groups were not significantly different in a number of variables - that is, age, sex, operator grade, length to first postoperative visit, pre and postoperative visual acuities, anaesthetic type, and presence of diabetes mellitus. As the groups are similar on a significant number of variables it suggests that comparison of complication rate is a valid one.

There was one case of iris prolapse in each group, each of which presented at the first postoperative visit. There were no wound leaks otherwise missed in either group before discharge implying that wound leaks may be effectively screened for at the 4-6hour review. There were no cases of endophthalmitis in this series in the time to the first postoperative visit. However, there was one delayed case in the nonday-case group at 3 months after operation and 1 week after suture removal. We had no cases of early onset endophthalmitis out of 375 consecutive cases, giving an observed rate of less than $0 \cdot 13 \%(0.5 / 375)$ and, assuming a Poisson distribution when the variance is equal to the mean, the upper limit is still less than $0.5 \%$ which is in keeping with published rates of $0 \cdot 1-0 \cdot 4 \% .^{67}$

We have no reason to suspect that same day discharge should result in an increase in the endophthalmitis rate but there is a concern that its detection may be delayed. Endophthalmitis due to the more virulent organisms typically presents at 48 to 72 hours $^{8}$ and only $10 \%$ of cases

Table 6 Number and type of complications in the 'true' day-case and non-day-case groups

\begin{tabular}{lcc}
\hline Complications & Day-case & Non-day-case \\
\hline Raised IOP & 3 & 5 \\
Wound leak & 3 & 4 \\
Corneal oedema & 2 & 2 \\
Vitreous haemorrhage & 1 & 1 \\
Iris prolapse & 1 & 1 \\
Late endophthalmitis & 0 & 1 \\
Total & 10 & 14 \\
\hline
\end{tabular}


present on the first postoperative day. ${ }^{9}$ Therefore, as a screening procedure for endophthalmitis the first postoperative day review is extremely inefficient. The expected number of cases to be detected is $10 \%$ of the observed rate of less than $0.13 \%$ which is less than 1 case per 7693 reviews. Instead, we suggest screening those patients who report symptoms (such as increasing pain or redness) by asking them to attend a casualty department as an emergency and limit routine review to those patients who are unable to report such symptoms (for example, patients with dementia).

There was no excess morbidity in this study period between the two groups. The ability to dispense with the first day postoperative review is of clear practical benefit for many patients and this study suggests that this is a safe practice for selected patients.
1 Chisholm JJ. The rational method of treating cataract patients to the exclusion of compresses, bandages and dark rooms. Am $\mathcal{F}$ Ophthalmol 1886; 3: 135-40.

2 Christy NE. Effect of early ambulation on the incidence of postoperative complications of cataract surgery. $A m \mathcal{F}$ Ophthalmol 1960; 49: 293-7.

3 Galin MA, Boniuk V, Obstbaum S, Glasser M. Outpatient cataract surgery. Trans Ophthalmol Soc UK 1975; 95: 42-5.

4 Jervey JW Jnr, Brown RA. Cataract surgery as an outpatient Jervey JW Jnr, Brown RA. Cataract surgery as an
procedure. Am $\mathcal{F}$ Ophthalmol 1963; 56: 58-63.

5 Kanski JJ. Cataract surgery. In: Miller S, ed. Clinical ophthalmology. London: Butterworths, 1987: 282-91.

6 Katten HM, Flynn HW, Pflugfelder SC, Roleston C, Forster RK. Nosocomial endophthalmitis survey: current incidence of infection after intraocular surgery. Ophthalmology 1991; 98: 227-38.

7 Meinkoff JA, Speaker MG, Marmor M, Raskin EM. A case control study of risk factors for post-operative endophthalmitis. Ophthalmology 1991; 98: 1761-8.

8 D'Amico DJ, Noorily SW. Postoperative endophthalmitis. In: Albert DM, Jakobiec FA, eds. Principles and practice of In: Albert DM, Jakobiec FA, eds. Principles and practice of Saunders, 1994; Vol 2: 1159-69.

9 Fish A, Salvanet A, Prazuck T, Forestier F, Gerband K Coscas G, et al, and the French Collaborative Study Group in Endophthalmitis. Epidemiology of infective endophthalmitis in France. Lancet 1991; 338: 